ORIGINAL ARTICLE

\title{
Effects of brief yoga exercises and motivational preparatory interventions in distance runners: results of a controlled trial
}

\author{
B Donohue, A Miller, M Beisecker, D Houser, R Valdez, S Tiller, T Taymar
}

Br J Sports Med 2006;40:60-63. doi: 10.1136/bjsm.2005.020024

See end of article for authors' affiliations

\section{Correspondence to:} Dr Donohue, University of Nevada, 4505 Maryland Parkway, Las Vegas, NV 89154,USA; donohueb@ unlv.nevada.edu

Accepted 12 July 2005

\begin{abstract}
Objective: To examine the efficacy of two preparatory interventions on one mile run performance in 90 high school long distance runners.

Method: After participants had completed a one mile baseline run, they were randomly assigned to participate in either one of two interventions (brief yoga exercises, motivational shouting exercises) or a no intervention control condition. Experimental conditions were implemented one week after the baseline run about 20 minutes before a second one mile trial.

Results: Participants assigned to the motivational intervention improved their running performance significantly more than those assigned to the other two conditions. Although the magnitude of the effect was small, participants assigned to yoga exercises showed significant improvements in running performance relative to control condition participants. Consumer satisfaction ratings indicated that participants who were assigned to the motivational and yoga exercise groups liked their interventions more than those assigned to the control group.

Conclusion: Motivational and yoga interventions designed to improve long distance running performance were equally acceptable to the participants, but the former had a greater effect.
\end{abstract}

$\mathrm{P}$ ositive self statements have been found to improve performance in athletic tasks by enhancing physiological preparation, ${ }^{12}$ inspiring greater effort, and creating positive mood and confidence. ${ }^{3}$ Pre-event motivationally based interventions have shown effectiveness in controlled intervention trials. ${ }^{4-10}$ However, most of the mental preparation interventions have been evaluated in contrived sport situations with nonathlete samples, leaving a void in the literature about which preparation strategies are best for particular athletic events. ${ }^{6}$

Specific to long distance running performance, two controlled intervention outcome studies have shown the effectiveness of pre-event interventions that use athlete generated motivational statements. The first study ${ }^{4}$ involved six National Collegiate Athletic Association division one female cross country distance runners. A Latin squares experimental design was used to evaluate effectiveness of two interventions, as compared with an attention control condition. The results indicated that verbal instructions focusing on specific running techniques five minutes before running were slightly more effective than motivational statements made during the same time frame, and that both of these interventions led to greater improvement in run performance than the attention control.

Building on results of the previous study, Miller and Donohue $^{6}$ examined the effects of combining running technique instructions and motivational statements on long distance run performance in 90 high school cross country runners. After participants had completed a one mile baseline run, they were randomly assigned to receive one of three experimental conditions three minutes before a second one mile trial runthat is, listening to a personalised script of motivational and running technique statements on headphones, listening to music on headphones, listening to no sound on headphones. Participants chose their own motivational song, or were assisted in choosing their own motivational/running technique statements from a generic list, depending on the respective intervention received. The results indicated that participants assigned to the motivational and running technique statements, as well as the music intervention, significantly improved their run performance, whereas participants in the no-sound control condition did not. Therefore, preliminary evidence suggests listening to motivational statements before long distance running may enhance performance.

The use of yoga has recently gained popularity in preparing athletes for competition. As reviewed by Ives, ${ }^{11}$ use of yoga exercises has been shown to positively influence flexibility, ventilation, hypertension, mood, breathing regulation, and relaxation, all of which have been indicated in the improvement of run performance. In an uncontrolled trial, participants who practiced yoga for six weeks showed improvements in anaerobic capacity which was due to increased NAD. ${ }^{12}$ However, we could find no controlled outcome studies of yoga in the improvement of athletic performance. Thus, although yoga appears to be promising in the improvement of athletic performance, the drawing of any definitive conclusions is prohibited at this time.

The purpose of this study was to use controlled methodology in (a) determining the relative effects of preparatory motivational and abbreviated yoga exercises in the improvement of one mile run performance in high school distance runners, and $(b)$ evaluating the social validity of these interventions-that is, consumer satisfaction.

\section{METHOD}

\section{Participants}

Participants were 90 high school distance runners (49 male, 41 female) from five high schools in predominantly middle income communities in southern Nevada. They ranged in age from 14 to 18 years (mean (SD) 15.7 (1.1)), and most were high school sophomores and juniors (mean (SD) grade 10.34 (1.0)). The racial breakdown was $79 \%(\mathrm{n}=71)$ white, $7 \%$ (n $=6)$ Hispanic, $7 \%$ African-American, $2 \%(n=2)$ AsianAmerican, and the remaining $6 \%(\mathrm{n}=5)$ other ethnic minorities. Before this study, the mean (SD) $1600 \mathrm{~m}$ (one mile) personal record (minutes:seconds) was 5:27 (0:45.10) for male participants and 6:1 (0:38.2) for female participants.

\section{Procedures}

Recruitment of participants

A research assistant informed five local high school track and cross country head coaches of the study, and these coaches 
invited their long distance runners to participate in the study. All interested youngsters (about $95 \%$ of those invited to participate), with consent of their parents, assented to be participants in this study after being informed of its nature, including the benefits and risks of participation and confidentiality.

\section{Assessment of background information}

All potential subjects were asked to provide basic characteristics (sex, age, ethnicity) and background information about their cross country and track experience (personal best $1600 \mathrm{~m}$ run time, preliminary ratings of how much the athlete expected participating in motivational/high arousal group interventions and yoga/low arousal group interventions to influence run performance).

\section{Baseline run}

After providing background information, participants were instructed to run a one mile trial on a $400 \mathrm{~m}$ track to the "best of their ability" after completing their typical 20 minute warm up exercise routine- that is, stretching, running strides.

\section{Assessment of motivational statements}

Immediately after completing the baseline trial run, participants were given a list of 40 motivational statements, such as "you're going to dominate today", that have been used in previous intervention outcome studies. ${ }^{4}{ }^{6}$ Participants rated each motivational statement on a five point Likert scale $(1=$ not at all motivating, 5 = extremely motivating). Each participant could also add, and subsequently rate using the same five point scale, his or her own self generated motivational statements. For each participant, their most preferred motivational statement was selected for use if assigned to the motivational group intervention scheduled to occur one week later.

\section{Random assignment to intervention groups}

After the baseline run, participants were grouped into sets of three according to similar one mile baseline trial times. The three participants in each set were then randomly assigned to one of three experimental conditions scheduled to occur 20 minutes before the running of a second one mile trial on the same track one week after the baseline trial. The experimental conditions were: (a) participation in a motivational group intervention; $(b)$ participation in a yoga group intervention; (c) participation in an attention control group intervention.

\section{Experimental group conditions}

The motivational intervention consisted of first distributing a note card to each participant. Each note card included the participant's most preferred motivational statement selected from the list of 40 statements given the previous week. The participants then formed a circle and, for about nine minutes, were instructed to shout out their chosen motivational statement, one person at a time in a clockwise fashion. Next, participants played a game in which they took turns rolling a large ball to another participant after shouting the motivational statement listed on the note card-for example, "you're the definition of speed". Each participant rolled the ball and shouted a motivational statement at least three times. The participants were then instructed to form two parallel lines of about $50 \mathrm{~m}$ length - that is, participants were evenly spaced. While in the line, all participants shouted motivational statements to participants who strided between the two lines for the $50 \mathrm{~m}$ distance-that is, all participants who were assigned to this condition completed two $50 \mathrm{~m}$ strides. The strides lasted for about eight minutes. Lastly, the participants assembled into a tightly formed circle, and were instructed to enthusiastically and loudly shout their motivational statements for about three minutes. Facilitators encouraged athletes to shout these statements spontaneously, and randomly.

The brief yoga intervention consisted of 11 basic entry level yoga asana positions: mountain pose, asana 2, forward bend, lunge, plank, staff, upward facing dog, downward facing dog, lunge return, mountain pose return, and breath of fire. ${ }^{13}$ In this intervention, one facilitator provided verbal detailed instructions for each position, and another facilitator modelled each posture to the participants. The intervention lasted 20 minutes.

The attention control condition included a facilitator asking participants questions relevant to competitive running-for example, how they were feeling, what events they run particularly well. While the questions were asked, participants were instructed to engage in their typical warm up routine for 20 minutes.

\section{Trial run after the intervention}

After the respective interventions had been performed, participants were permitted a few minutes to complete their typical warm up routine, such as final stretches and strides, before they were instructed to participate in a one mile trial run (same track as the baseline trial). For each high school, the baseline and post-intervention trials occurred at the same time of day. The trial runs were scheduled so that officially sanctioned track meets occurred more than two weeks preceding the baseline trial and more than two weeks after the post-intervention trial. Attempts were made to keep both trial runs competitive, including instructions to run their best and informing participants that both would be timed.

\section{Consumer satisfaction questionnaire after the intervention}

On completion of the second one mile trial run, all participants were instructed to complete a two item consumer satisfaction questionnaire. One question asked the participants to rate how much they believed that the intervention had improved their performance $(1=$ no improvement at all, $5=$ extremely improved), and a second question asked the participants to rate their satisfaction with the intervention $(\mathrm{l}=$ not at all satisfied, $5=$ extremely satisfied). Participants were not informed of their running times before completing the questionnaire.

\section{RESULTS}

\section{Protocol adherence}

To maintain integrity of the motivational and yoga interventions, several strategies were used ${ }^{14}$ including use of protocol checklists by facilitators that dictate task specific prevention procedures and continuing supervision and corrective feedback to facilitators.

Protocol checklists were used to obtain reliability and validity estimates for the motivational and yoga group interventions. Specifically, facilitators indicated on the respective protocol checklist whether each task was performed. Independent raters trained in the respective interventions, and blinded to the nature of the study, observed the groups and independently indicated on a separate checklist whether each task in the protocol had been completed. The completed facilitators' and raters' lists were compared, and a reliability estimate was computed. Reliability was calculated by dividing the total number of agreements by the total number of agreements plus disagreements, and multiplying the resulting dividend by 100 . The validity estimate was based only on the lists completed by facilitators, and was calculated by dividing the number of tasks completed by the total number of possible tasks, and multiplying the dividend by 100 . For both interventions, the reliability estimate was $100 \%$, and the validity estimate was $100 \%$. Thus the facilitators in both intervention groups indicated that they strictly adhered to their respective protocol, and protocol adherence was corroborated by independent raters who were blinded to the nature of this study. 
Table 1 Baseline and post-intervention one mile trial run times (minutes:seconds)

\begin{tabular}{|c|c|c|c|c|c|c|c|}
\hline \multirow[b]{2}{*}{ Intervention } & \multirow[b]{2}{*}{ No } & \multicolumn{2}{|c|}{ Baseline } & \multicolumn{2}{|c|}{ Post-intervention } & \multicolumn{2}{|c|}{ Improvement } \\
\hline & & Mean & SD & Mean & SD & Mean & SD \\
\hline Attention control & 29 & $6: 39$ & $0: 54$ & $6: 40$ & $0: 54$ & $-0: 01$ & $0: 54$ \\
\hline Yoga & 31 & $6: 20$ & $0: 49$ & $6: 19$ & $0: 49$ & 0:01 & $0: 49$ \\
\hline Motivational statements & 30 & $6: 07$ & $0: 59$ & $6: 02$ & $0: 58$ & $0: 05$ & $0: 59$ \\
\hline
\end{tabular}

\section{Comparability of groups before the intervention}

To assess the comparability of the three experimental groups, a two tailed, one way analysis of variance was conducted on age, and $\chi^{2}$ tests were performed on ethnicity and sex, with the type of intervention received serving as the independent variable. The two tailed $\chi^{2}$ tests indicated that the experimental groups were not significantly different with respect to the number of male and female participants and ethnic composition (both $\mathrm{p}>0.05$ ). The analysis of variance revealed a significant difference between experimental conditions with respect to age $(F=3.33, \mathrm{df}=2,86, \mathrm{p}<0.05)$.

To assess comparability of the experimental groups on one mile baseline trial performance, analysis of variance was conducted using the type of intervention received as the independent variable, and time to run the one mile baseline trial as the dependent variable. Results indicated that participants in the experimental groups did not differ with respect to their baseline run performance $(p>0.05)$.

\section{Effectiveness of interventions}

For each of the experimental conditions, table 1 presents the baseline and post-intervention trial run times. To examine the influence of the interventions on trial run performance, a repeated measures multivariate analysis of covariance was performed. The independent variable was type of intervention received-that is, motivational statements, yoga, or attention control. The repeated dependent measure was run performance (time to run one mile in the baseline and post-intervention trials). Age was selected as a covariate because the experimental groups were found to differ with respect to age.

The multivariate analysis of covariance indicated a significant main effect for time. However, a significant time by intervention group interaction effect was found (Wilkes $\lambda$ $=0.943, \mathrm{~F}=5.17 \mathrm{df}=1,85, \mathrm{p}<0.001)$. Post hoc, one tailed, independent samples $t$ tests were conducted to identify which interventions led to greatest relative improvement in run performance. The mean differences between baseline and post-intervention scores were computed, and three independent samples $t$ tests were conducted using the difference scores as the dependent variable, and the intervention received as the independent variable. Participants who received the motivational group intervention showed significantly greater improvement in run performance than those who received the yoga intervention $(t=5.16 \mathrm{df}=$ $1,59, \mathrm{p}<0.001)$ and those who received the control condition $(t=5.88, \mathrm{df}=1,57, \mathrm{p}<0.001)$. Participants in the yoga intervention showed significantly greater improvements in run performance than those who were assigned to the control condition $(t=2.17$, df $=1,58, \mathrm{p}<0.05)$.

\section{Consumer satisfaction and participant perceptions of intervention outcome}

Table 2 presents the participant responses to the two five point Likert items reflecting perceived improvement in run performance of the intervention received and personal satisfaction with the respective intervention. A two tailed, one way analysis of variance indicated that participants in the three experimental groups differed in their degree of perceived improvement $(F=36.61, \mathrm{df}=2,87, \mathrm{p}<0.001)$ and personal satisfaction $(F=36.66, \mathrm{df}=2,87, \mathrm{p}<0.001)$ with the interventions. Post hoc, one tailed, independent samples $t$ tests indicated that participants who received the attention control condition endorsed significantly lower ratings in perceived intervention improvement than participants who received the motivational group intervention $(t=$ 8.73, $\mathrm{df}=1,57, \mathrm{p}<0.001)$ and yoga intervention $(t=4.25$, $\mathrm{df}=1,58, \mathrm{p}<0.001)$. Participants who received the yoga exercises endorsed significantly lower ratings in perceived improvement than participants in the motivational group intervention $(t=4.33$, df $=1,59, \mathrm{p}<0.001)$.

Perceived improvement and satisfaction were evaluated using Likert scales: 1, no improvement or no satisfaction; 5, extremely improved or extremely satisfied.

Similarly, one tailed, independent samples $t$ tests indicated that participants who received the control condition endorsed significantly lower ratings in perceived intervention satisfaction than those who received the motivational group intervention $(t$ $=8.91, \mathrm{df}=1,57, \mathrm{p}<0.001)$ and yoga intervention $(t=6.19$, $\mathrm{df}=1,58, \mathrm{p}<0.001)$. The mean intervention satisfaction scores were similar between participants assigned to the brief yoga and motivational group interventions. Thus perceptions of run performance improvement were consistent with objective timed improvements in run performance. However, satisfaction scores indicated that the yoga and motivational interventions were equally more liked than the control condition.

\section{DISCUSSION}

The results of this study indicate that a motivational preparatory group intervention significantly improved one mile run performance more than attention control and brief yoga exercise interventions. Briefly implementing the yoga exercises immediately before running appeared to significantly enhance run performance relative to the control group, albeit the magnitude of this effect was small. Of course, the potential

\begin{tabular}{|c|c|c|c|c|c|}
\hline \multirow[b]{2}{*}{ Intervention } & \multirow[b]{2}{*}{ No } & \multicolumn{2}{|c|}{ Improvement } & \multicolumn{2}{|c|}{ Satisfaction } \\
\hline & & Mean & SD & Mean & SD \\
\hline Attention control & 29 & 1.86 & 0.99 & 2.00 & 0.96 \\
\hline Yoga & 31 & 2.94 & 0.96 & 3.71 & 1.16 \\
\hline Motivational statements & 30 & 3.93 & 0.83 & 4.10 & 0.85 \\
\hline
\end{tabular}


What is already known on this topic

- Motivationally based preparatory interventions have been found to improve performance in athletic tasks

- However, most of the mental preparation interventions have been evaluated in contrived sport situations with non-athlete samples, so it is not known which preparation strategies are best for particular athletic events

efficacy of other forms or durations of yoga in the enhancement of long distance running performance cannot be inferred from this study. Indeed, it may be that the implementation of yoga exercises across several weeks for extended time periods may be more effective than the abbreviated yoga exercise programme used in this study to provide a temporally equivalent comparison to assist in evaluating the motivational intervention. Indeed, the yoga condition in this study acts as a viable attention control for variables that may have affected running performance-that is, training, illness, feedback from others.

Participant perceptions of performance outcome were consistent with the aforementioned results. Participants who were assigned to the motivational and yoga conditions liked their respective interventions more than those in the control group, whereas yoga and motivational group interventions were liked about the same. The latter findings support the social validity of the motivational and yoga interventions, suggesting that other long distance high school runners may find these approaches worthy of adoption. However, given the difference in improved run performance between the brief yoga and motivational interventions, the motivational group intervention is clearly recommended.

The developed motivational intervention appears to be fun, and is consistent with contemporary motivational strategies implemented in team sports, but until this study were not empirically validated-for example, team jumps and down in a tight circle yelling motivational statements before a basketball or football game. The efficacy of the motivational group intervention shown in this study is also consistent with the results of other outcome evaluations involving athletic mental preparation interventions. For instance, the controlled study of Theodorakis et $a l^{9}$ indicated that endurance tasks were positively influenced by motivational self statements. Donohue et al ${ }^{4}$ found that having a facilitator deliver motivational statements to cross country runners before they ran $1000 \mathrm{~m}$ appeared to be more effective than an attention control in improving running performance. Miller and Donohue $^{6}$ also showed that the running performance of cross country runners benefited after they had listened to motivational statements played on a compact disc. The latter intervention is practical because all members of the cross country team can benefit from using their own personalised motivational compact disc. Similarly, the motivational intervention developed in this study may be used to accommodate a team of cross country runners and is cost effective.

Although attempts were made in this study to create a competitive environment in which to evaluate performance, it should be stressed that the trials were contrived. It is probable that the motivational intervention developed would be effective in actual track meet situations and perhaps other team sporting events, such as baseball, basketball, and volleyball. Indeed, the motivational intervention may be enhanced in track meets, as motivation is particularly relevant to performance in competitive situations. However, such generalisation is not possible given the inherent problems in the present methodology. Thus future studies will need to evaluate the developed motivational group intervention in competitive situations.
What this study adds

- This study shows the merits of a brief motivationally based preparatory intervention in enhancing run performance in distance runners

- This is one of the very few controlled trials to show the importance of using motivational statements immediately before long distance running

\section{Authors' affiliations}

B Donohue, A Miller, M Beisecker, D Houser, R Valdez, S Tiller, T Taymar, University of Nevada, Las Vegas, NV, USA

Competing interests: none declared

\section{REFERENCES}

1 Desiderato O, Miller IB. Improving tennis performance by cognitive behavior and modification techniques. In: Martin GL, Hrycaiko D, eds. Behavior modification and coaching principle, procedures, and research. Springfield, IL: CC Thomas, 1979:293-5.

2 Weinberg RS, Smith J, Jackson A, et al. Effects of association, dissociation, and positive self-talk strategies on endurance performance. Canadian Journal of Applied Sport Science 1984;9:25-32.

3 Hardy L, Jones G, Gould D. Understanding psychological preparation for sport: theory and practice. Chichester: Jones Wiley \& Sons, 1996.

4 Donohue B, Barnhart R, Covassin T, et al. The development and initial evaluation of two promising mental preparatory methods in a sample of female cross-country runners. Journal of Sport Behavior 2000;24:2-12.

5 Gould D, Weinberg R, Jackson A. Mental preparation strategies, cognitions, and strength performance. Journal of Sport Psychology 1980;2:329-39.

6 Miller A, Donohue B. The development and controlled evaluation of athletic mental preparation strategies in high school distance runners. Journal of Applied Sport Psychology 2003;15:321-34.

7 Rushall BS, Hall M, Roux L, et al. Effects of three types of thought content instructions in skiing performance. The Sport Psychologist 1988;2:283-97.

8 Shelton AO, Mahoney MJ. The content and effect of "psyching-up" strategies for weight lifters. Cognit Ther Res 1978;2:275-84.

9 Theodorakis $Y$, Weinerg $R$, Natsis $P$, et al. The effects of motivational versus instructional self-talk on improving motor performance. The Sport Psychologist 2000;14:263-72.

10 Weinberg RS, Gould D, Jackson A. Cognition and motor performance: effect of matching-up strategies on three motor tasks. Cognit Ther Res 1980;4:239-45.

11 Ives JC. Beyond mind-body exercise hype. Phys Sportmed 2000;28:67-81.

12 Pansare MS, Kulkarni AN, Pendse UB. Effect of yogic training on serum LDH levels. J Sports Med Phys Fitness 1989;29:177-8.

13 Lark L. Power yoga for beginners. Scranton, PA: Harper Collins, 2003.

14 Yeaton WH, Schrest L. Critical dimensions in the choice and maintenance of successful treatment: strength, integrity, and effectiveness. I Consult Clin Psychol $1981 ; 49: 156-67$.

\section{COMMENTARY}

The key finding in this paper is that the intervention groups clearly preferred a pre-race intervention that helped to motivate or prepare them for their effort. The high perceived satisfaction with either yoga or motivational preparation addresses a key concern of coaches: "how do you best prepare an athlete to achieve a peak performance in a given race." Work in sports psychology on the concept of "flow" (Jackson, Csikszentmihalyi, and others) points to athlete satisfaction and happiness as key components to achieving success. I am not impressed that this trial clearly showed improved performance in the yoga and motivational preparation groups, because of the many possible variables. However, if the athletes' satisfaction clearly improved this much, additional studies may clarify the performance result.

K B Fields Moses Cone Health System, Greensboro, NC, USA; bert.fields@mosescone.com 